\title{
An Introduction to Lie Groups
}

\author{
Amor Hasić \\ Department of Mathematics, Faculty of Natural Sciences and Mathematics, University of Montenegro, Podgorica, Montenegro \\ Email: amorhasic@gmail.com
}

How to cite this paper: Hasić, A. (2020) An Introduction to Lie Groups. Advances in Linear Algebra \& Matrix Theory, 10, 35-51.

https://doi.org/10.4236/alamt.2020.103004

Received: July 19, 2020

Accepted: September 27, 2020

Published: September 30, 2020

Copyright $\odot 2020$ by author(s) and Scientific Research Publishing Inc. This work is licensed under the Creative Commons Attribution International License (CC BY 4.0).

http://creativecommons.org/licenses/by/4.0/

\begin{abstract}
This paper is made out of necessity as a doctoral student taking the exam from Lie groups. Using the literature suggested to me by the professor, I felt the need to, in addition to that literature, and since there was more superficial in that book with some remarks about the examples given in relation to the left group. I decided to try a little harder and collect as much literature as possible, both for the needs of me and the others who will take after me. Searching for literature in my mother tongue I could not find anything, in English as someone who comes from a small country like Montenegro, all I could find was through the internet. I decided to gather what I could find from the literature in my own way and to my observation and make this kind of work. The main content of this paper is to present the Lie group in the simplest way. Before and before I started writing or collecting about Lie groups, it was necessary to say something about groups and subgroups that are taught in basic studies in algebra. In them I cited several deficits and an example. The following content of the paper is related to Lie groups primarily concerning the definition of examples such as The General Linear Group $G L(n, R)$, The Complex General Linear Group GL(n, C), The Special Linear Group $S L(n, R)=S L(V)$, The Complex Special Linear Group $S L(n, C)$, Unitary and Orthogonal Groups, Symplectic Group, The groups $R^{*}, C^{*}, S^{1}$ and $R^{n}$ and others. In addition, invariant vector fields and the exponential map and the lie algebra of a lie group. For me, this work has the significance of being useful to all who need it.
\end{abstract}

\section{Keywords}

Groups, Subgroups, Lie Groups, Invariant Vector Fields,

The Exponential Map

\section{Introduction}

A Lie group is, roughly speaking, an analytic manifold with a group structure such that the group operations are analytic. Lie groups arise in a natural way as 
transformation groups of geometric objects. Lie groups are not linear they are curved manifolds. Nevertheless, Lie's theorem reduces many questions about Lie groups to questions about Lie algebras. Questions about curved manifolds turn out to be equivalent to questions about linear algebra. This is a profound simplification, and it leads to a very rich theory. The theory of Lie groups answers these questions by replacing the notion of a finitely generated group with that of a Lie group - a group which at the same time is a finite-dimensional manifold. It turns out that in many ways such groups can be described and studied as easily as finitely generated groups-or even easier.

Lie groups and Lie algebras, together called Lie theory, originated in the study of natural symmetries of solutions of differential equations. However, unlike say the finite collection of symmetries of the hexagon, these symmetries occurred in continuous families, just as the rotational symmetries of the plane form a continuous family isomorphic to the unit circle. The theory as we know it today began with the ground-breaking work of the Norwegian mathematician Sophus Lie, who introduced the notion of continuous transformation groups and showed the crucial role that Lie algebras play in their classification and representation theory. Lie's ideas played a central role in Felix Klein's grand "Erlangen program" to classify all possible geometries using group theory. Today Lie theory plays an important role in almost every branch of pure and applied mathematics, is used to describe much of modern physics, in particular classical and quantum mechanics, and is an active area of research [1].

The General Linear Group $G L(n, R)$ is the group of invertible $n \times n$ matrices with real entries under matrix multiplication. This forms a group, because the product of two invertible matrices is again invertible, and the inverse of an invertible matrix is invertible, with identity matrix as the identity element of the group. The group is so named because the columns of an invertible matrix are linearly independent, hence the vectors/points they define are in general linear position, and matrices in the general linear group take points in general linear position to points in a general linear position. https://en.wikipedia.org/wiki/General_linear_group

The special linear group $S L(n, F)$ of degree $n$ over a field $F$ is the set of $n \times n$ matrices with determinant 1 , with the group operations of ordinary matrix multiplication and matrix inversion. This is the normal subgroup of the general linear group given by the kernel of the determinant

$$
\text { det }: G L(n, F) \rightarrow F^{X}
$$

where we write $F^{x}$ for the multiplicative group of $F$ (that is, $F$ excluding 0 ).

These elements are "special" in that they form a subvariety of the general linear group-they satisfy a polynomial equation (since the determinant is polynomial in the entries). https://en.wikipedia.org/wiki/Special_linear_group

The orthogonal group in dimension $n$, denoted $O(n)$, is the group of distance-preserving transformations of a Euclidean space of dimension $n$ that preserve a fixed point, where the group operation is given by composing transfor- 
mations. An orthogonal group is sometimes called the general orthogonal group, by analogy with the general linear group. Equivalently, it is the group of $n \times n$ orthogonal matrices, where the group operation is given by matrix multiplication; an orthogonal matrix is a real matrix whose inverse equals its transpose. The orthogonal group is an algebraic group and a Lie group. It is compact.

The orthogonal group in dimension $n$ has two connected components. The one that contains the identity element is a subgroup, called the special orthogonal group, https://en.wikipedia.org/wiki/Orthogonal_group.

The unitary group of degree $n$, denoted $U(n)$, is the group of $n \times n$ unitary matrices, with the group operation of matrix multiplication. The unitary group is a subgroup of the general linear group $G L(n, C)$. Hyperorthogonal group is an archaic name for the unitary group, especially over finite fields. For the group of unitary matrices with determinant 1 , see Special unitary group.

The purpose of researching this paper is, as I have already stated in the summary, to gather as much information as possible and introduce it into the basic meaning of Lie groups. That everyone can benefit from this work at the beginning and find guidelines for further study of Lie groups. To get the most important information through this work without having to use a lot of literature like me and to get information in an easier way.

\section{Groups, Subgroups, Definitions and Examples}

Definition 1: Group is a set $G$ together with a map $\mu: G \times G \rightarrow G$; $(x, y) \rightarrow x y$ and an element $e \rightarrow e_{G}$; such that the following conditions are fulfilled

1) An associative algebra is algebra $A$ whose associative rule is associative: $x(y z)=(x y) z$ for all $x, y, z \in G$

2) There exists an element $e \in G$, such that for all $x \in G$ we have $x * e=e * x=x$. Such an element $e \in G$ is called an identity in $G$.

3) For every $x \in G$ there exists an element $x^{-1} \in G$ such that $x * x^{-1}=x^{-1} x=x$ is called an inverse of a in $G$.

Definition 2: If in the group $(G ; \cdot)$ for all $x, y \in G$ is

$$
x \cdot y=y \cdot x
$$

Then we say that this structure is commutative (or Abel's), so we can speak of a commutative group.

Example 1: Let $(G, \cdot)$ be a group in which $x^{2}=e$ holds for each element a. We claim that is then $G$ a commutative group. Namely,

$$
\begin{gathered}
(x y)(x y)=e=x x=x e x=x(y y) x=(x y)(y x) /(x y)^{-1} \\
\Rightarrow x y=y x
\end{gathered}
$$

Example 2: If $(V ;+; \cdot)$ is a vector space, then $(V ;+)$ is an Abel group.

Definition 3: Let $S=\{e\}$ be a one-membered set and define a binary operation on $S$ by the formula $e \cdot e=e$. Then $(\{e\} ; \cdot)$ is a group we call a trivial group.

Definition 4: Subgroup of $G$ is a subset $H \subset G$ such that 
1) $e_{H} \in H$;

2) $x y \in H$ for all $x \in H$ and $y \in H$;

3) $x^{-1} \in H$ for every $x \in H$.

Definition 5: [2] nonempty subset $H$ of a group $G$ is a subgroup of $G$ if $H$ is a group under the same operation as $G$. We use the notation $H \subset G$ to mean that $H$ is a subset of $G$, and $H \leq G$ to mean that $H$ is a subgroup of $G$. For a group $G$ with identity element $e,\{e\}$ is a subgroup of $G$ called the trivial subgroup. For any group $G, G$ itself is a subgroup of $G$, called the improper subgroup. Any other subgroup of $G$ besides the two above is called a nontrivial proper subgroup of $G$.

Definition 6: Let $(G, \cdot)$ and $(H, *)$ groups. Function $f: G \rightarrow H$ is a homomorphism if for all $x, y \in G$ is valid:

1) $f\left(e_{G}\right)=e_{H}$

2) $f(x y)=f(x) * f(y)$ for all $x, y \in H$

Proof: a) If $x \in G, f(x)=f\left(x e_{G}\right)=f(x) f\left(e_{G}\right)$ and $H$ is group, hitting the left with $f(x)^{-1}, f\left(e_{G}\right)=e_{H}$, so $f(x)^{-1}=f\left(x^{-1}\right)$.

Definition 7: If $f: G \rightarrow H$ is a homomorphism. The kernel of $f$ defined by

$$
\text { kerf }=f^{-1}\left(e_{H}\right)=\left\{f(x)=e_{H}\right\}
$$

is also readily seen to be a subgroup of $G$.

Proof. We have to show that the kernel is non-empty and closed under products and inverses. Note that $\varphi(e)=f$. Thus Kerf is certainly non-empty. Now suppose that a and bare in the kernel, so that

$$
\begin{gathered}
f(x)=f(y)=\varphi . \\
f(x y)=f(x) \varphi(y)=\varphi \varphi=\varphi .
\end{gathered}
$$

Thus $x y \in \operatorname{Kerf}$ and so the kernel is closed under products. Finally suppose that $f(x)=e$. Then $f\left(x^{-1}\right)=f(x)^{-1}=\varphi$. Thus the kernel is closed under inverses, and the kernel is a subgroup.

Example 3: Let $R^{+}$denote the set of positive real numbers. Then there is the function $\ln : R^{+} \rightarrow R$ is a homomorphism of a group $\left(R^{+}, \cdot{ }^{-1} ; 1\right)$ into a group $(R,+,-, 0)$.

$$
\begin{gathered}
\ln \left(x^{-1}\right)=-\ln x \text { and } \ln 1=0 \\
\ln (x y)=\ln (x)+\ln (y)
\end{gathered}
$$

Example 4: Conjugation, i.e., function $g: C \rightarrow C$, given by $g(z)=\bar{z}$, represents the automorphism of the algebra $C=(C,+, \cdot, 0,1)$

Function $f \square \rightarrow H$ is a homomorphism

- Subjective group homomorphism is called an epimorphism

- Injective group homomorphism is called a monomorphism.

- Bijective group homomorphism is called an isomorphism.

- If $A=B$, we say that $x$ is an endomorphism.

- If $f$ is an isomorphism and an endomorphism, we say that $x$ is an automorphism. 
- Automorphism of $G$ we mean an isomorphism of $G$ onto itself.

Definition 9: The map $f \square \rightarrow H$ is called an isomorphism and $G$ and $H$ are said to be isomorphic if

1) $f$ is a homomorphism.

2) $f$ is a bijection.

- If $G$ is a group and $x \in G$; then the map $\ell_{x}: G \rightarrow G, y \rightarrow x y$; is called left translation by $x$.

- If $x \in G$; then $r_{x}: G \rightarrow G, y \rightarrow y x$ is called right translation by $x$

- If $x \in G$; then $C_{x}: G \rightarrow G, y \rightarrow x y x^{-1}$ is called conjugation by $x$.

\section{LIE GROUPS, DEFINITION AND EXAMPLES}

$A$ Lie group is a smooth (i.e., C 1 ) manifold $G$ equipped with a group structure so that the maps

$$
\begin{gathered}
\mu: G \times G \rightarrow G \\
\mu:(x y) \rightarrow x y
\end{gathered}
$$

and the inversion map

$$
\begin{gathered}
\imath: G \times G \rightarrow G \\
\imath: x \rightarrow x^{-1}
\end{gathered}
$$

are smooth.

In other words, the coordinates of the product must be differentiable functions of the coordinates of factors, and the coordinates of the inverse element must be differentiable functions of the coordinates of the element itself.

A Lie group over $\mathbb{C}$ is also called a complex Lie group and a Lie group over $\mathbb{R}$ is called a real Lie group. Any complex Lie group may be considered as a real Lie group of doubled dimension.

\section{Example 1:}

1) $R^{n}$ together with addition+ and the neutral element 0 is a Lie group.

2) $R^{*}=R \backslash\{0\} \quad$ is an open subset of $R$; hence a smooth manifold. Equipped with the ordinary scalar multiplication and the neutral element $1 ; R^{*}$ is a Lie group.

3) $C^{n}$ together with addition + and the neutral element 0 is a Lie group.

4) $C^{*}=C \backslash\{0\}$ is an open subset of $C$; hence a smooth manifold. Equipped with the ordinary scalar multiplication and the neutral element $1 ; C^{*}$ is a Lie group.

Example 2: Let

$$
G=R \times R \times S^{1}=\left\{(x, y, p) \mid x \in R, y \in R, p \in S^{1} \subset C\right\}
$$

Equipped with the group product given by

$$
\left(x_{1}, y_{1}, p_{1}\right) *\left(x_{2}, y_{2}, p_{2}\right)=\left(x_{1}+x_{2}, y_{1}+y_{2}, \mathrm{e}^{i x_{1} y_{2}} p_{1} p_{2}\right)
$$

Then $G$ is a Lie group.

[3] The General Linear Group $G L(n, R)$

Let $n$ be a positive integer, and let $M(n, R)$ be the set of real $n \times n$ matrices. 
Equipped with entry wise addition and scalar multiplication $M(n, R)$ is a linear space, which in an obvious way may be identified with $R^{n^{2}}$. For $A \in M(n, R)$ we denote by $A_{i j}$ the entry of $A$ in the $i$-th row and the $j$-th column.

The maps $\xi_{i j}: A \rightarrow A_{i j}$ may be viewed as a system of (linear) coordinate functions on $(n, R)$. In terms of these coordinate functions, the determinant function det: $M(n, R) \rightarrow R$ is given by

$$
\text { det }=\sum_{\sigma \in S_{n}} \operatorname{sgn}(\sigma) \xi_{1 \sigma(1)} \cdots \xi_{n \sigma(n)}
$$

where $S_{n}$ denotes the group of permutations of $\{1, \cdots, n\}$ and where sgn denotes the sign of a permutation. It follows from this formula that det is smooth.

The set $G L(n, R)$ of invertible matrices in $M(n, R)$ equipped with the multiplication of matrices, is a group. As a set it is given by

$$
G L(n, R)=\{A \in M(n, R) \mid \operatorname{det} A \neq 0\}
$$

Thus, $G L(n, R)$ is the pre-image of the open subset $R^{*}=R \backslash\{0\}$ of $R$ under det. As the latter function is continuous, it follows that $G L(n, R)$ is an open subset of $M(n, R)$. As such, it may be viewed as a smooth manifold of dimension $n^{2}$. In terms of the coordinate functions $\xi_{i j}$ the multiplication map $\mu: G L(n, R) \times G L(n, R) \rightarrow G L(n, R)$ is given by

$$
\xi_{k l}(\mu(A, B))=\sum_{i=1}^{n} \xi_{k i}(A) \xi_{i l}(B)
$$

It follows that $\mu$ is smooth. Given $A \in M(n, R)$ we denote by $A^{\mathrm{T}}$ the transpose of $A$. Moreover, for $1 \leq i, j \leq n \mathrm{n}$ we denote by $M_{i j}(A)$ the matrix obtained from $A$ by deleting the $i$-th row and $j$-th column.

The co-matrix of $A$ is defined by

$$
A_{i j}^{c o}=(-1)^{i+j} \operatorname{det} M_{i j}\left(A^{\mathrm{T}}\right)
$$

Clearly, the map $A \rightarrow A^{c o}$ is a polynomial, hence smooth map from $M(n, R)$ to itself. By Cramer's rule the inversion $\imath: G L(n, R) \rightarrow G L(n, R)$, $A \rightarrow A^{-1}$ is given by

$$
\iota(A)=(\operatorname{det} A)^{-1} A^{c o}
$$

It follows that $l$ is smooth, and we see that $G L(n, R)$ is a Lie group.

Obviously $G L(n, R)$ is an $n^{2}$-dimensional noncompact Lie group, and it is not connected. In fact, it consists of exactly two connected components,

$$
\begin{aligned}
& G L_{+}(n, R)=\{A \in M(n, R) \mid \operatorname{det} A>0\} \\
& G L_{-}(n, R)=\{A \in M(n, R) \mid \operatorname{det} A<0\}
\end{aligned}
$$

\section{[4]: The Complex General Linear Group $G L(n ; C)$}

This calls for some explanation, since $G L(n, C)$ is not a group of real matrices, as required by Definition:. A linear group is a closed subgroup of $G L(n, R)$. However, we can represent each complex matrix $Z \in M(n, C)$ by a 
real matrix $R Z \in M(2 n, R)$ in the following way. If we "forget" scalar multiplication by non-reals, the complex vector space $V=C^{n}$ becomes a real vector space $R V$ of twice the dimension, with basis

$(1,0, \cdots, 0),(i, 0, \cdots, 0),(0,1, \cdots, 0),(0, i, \cdots, 0), \cdots,(0,0, \cdots, 1),(0,0, \cdots, i)$ :

Moreover each matrix $Z \in M(n, C)$, i.e. each linear map

$$
Z: V \rightarrow V
$$

Defines a linear map

$$
R Z: R V \rightarrow R V
$$

i.e. a matrix $R Z \in M(2 n, R)$.

Concretely, in passing from $Z$ to $R Z$ each entry

$$
Z_{j, k}=X_{j, k}+i Y_{j, k}
$$

is replaced by the $2 \times 2$ matrix

$$
\left(\begin{array}{cc}
X_{j, k} & -Y_{j, k} \\
Y_{j, k} & X_{j, k}
\end{array}\right)
$$

The map

$$
Z \rightarrow R Z: M(n, C) \rightarrow M(2 n, R)
$$

is injective; and it preserves the algebraic structure, i.e.

- $R(Z+W)=R Z+R W$

- $R(Z W)=(R Z)(R W)$

- $R(a Z)=a(R Z), \forall a \in R$

- $R I=I$

- $R\left(Z^{*}\right)=(R Z)^{\prime}$

It follows in particular that $R Z$ is invertible if and only if $Z$ is; so $R$ restricts to a map

$$
Z \rightarrow R Z: G L(n, C) \rightarrow G L(2 n, R)
$$

Whenever we speak of $G L(n, C)$, or more generally of any group $G$ of complex matrices, as a linear group, it is understood that we refer to the image $R G$ of $G$ under this injection $R$.

The matrix $X \in G L(2 n, R)$ belongs to $G L(n, C)$ if is built out of $2 \times 2$ matrices of the form

$$
\left(\begin{array}{cc}
x & -y \\
y & x
\end{array}\right)
$$

This can be expressed more neatly as follows. Let

$$
i I \rightarrow J=\left(\begin{array}{ccccc}
0 & -1 & & & \\
1 & 0 & & & \\
& & 0 & -1 & \\
& & 1 & 0 & \\
& & & & \ddots
\end{array}\right)
$$

Since any scalar multiple of the identity commutes with all matrices, 


$$
X \in M(n, C) \Rightarrow(i I) X=X(i I)
$$

Applying the operator $R$,

$$
X \in R M(n, C) \Rightarrow J X=X J
$$

Conversely, if $J X=X J$ then it is readily verified that $X$ is of the required form.

Thus

$$
R M(n, C)=f\{X \in M(2 n, R): J X=X J\}
$$

and in particular

$$
G L(n, C)=\{T \in G L(2 n, R): J X=X J\}
$$

[3]: The Special Linear Group $S(n, R)=S L(V)$

Let $V$ be a finite dimensional real linear space. We define the special linear group

$$
S L(V)=\{A \in G L(V) \mid \operatorname{det} A=1\}
$$

Note that det is a group homomorphism from $G L(V)$ to $R^{*}$. Moreover, $S L(V)$ is the kernel of det. In particular, $S L(V)$ is a subgroup of $G L(V)$. We will show that $S L(V)$ is a sub-manifold of $G L(V)$ of co-dimension 1. Suffices to do this at the element $=I_{V}$.

Since $G=G L(V)$ is an open subset of the linear space End $(V)$ its tangent space $T_{I} G$ may be identified with $\operatorname{End}(V)$. The determinant function is smooth from $G$ to $R$ hence its tangent map is a linear map from End $(V)$ to $R$. Tangent map is the trace $\operatorname{tr}: \operatorname{End}(V) \rightarrow R ; A \rightarrow \operatorname{tr}(A)$. Clearly $\operatorname{tr}$ is a surjective linear map. This implies that det is submersive at I. That $S L(V)$ is a smooth co-dimension 1 sub-manifold at $I$ :

[4]. The Complex Special Linear Group SLn,

$$
S L(n, C)=\{A \in G L(n, C) \mid \operatorname{det} A=1\}
$$

Note that the determinant here must be computed in $M(n, C)$, not in $M(2 n, R)$.

Thus

$$
T=(i) \notin S L(1, C)
$$

Although

$$
R T=\left(\begin{array}{cc}
0 & -1 \\
1 & 0
\end{array}\right) \in S L(2, R)
$$

[5] Unitary and Orthogonal Groups

An $n \times n$ complex matrix $A$ is said to be unitary if the column vectors of $A$ are orthonormal, that is, if

$$
\sum_{i=1}^{n} \overline{A_{k l}} A_{k j}=\xi_{l j}
$$


We may rewrite as

$$
\sum_{i=1}^{n}\left(A^{*}\right)_{l k} A_{k j}=\xi_{l j}
$$

where $\xi_{l j}$ is the Kronecker delta equal to 1 if $l=j$ and equal to zero if $l \neq j$.

Here $A$ is the adjoint of $A$, defined by

$$
\left(A^{*}\right)_{l k}=\overline{A_{k l}}
$$

Equation says that $A^{*} A=I$; thus, we see that $A$ is unitary if and only if $A^{*}=A^{-1}$. In particular, every unitary matrix is invertible.

The adjoint operation on matrices satisfies $(A B)^{*}=B^{*} A^{*}$. from this, we can see that if $A$ and $B$ are unitary, then

$$
(A B)^{*}=B^{*} A^{*} A B=B^{-1} A^{-1} A B=I
$$

showing that $A B$ is also unitary. Furthermore, since $\left(A A^{-1}\right)^{*}=I^{*}=I$, we see that $\left(A^{-1}\right)^{*} A^{*}=I$, which shows that $\left(A^{-1}\right)^{*}=\left(A^{*}\right)^{-1}$. Thus, if $A$ is unitary, we have

$$
\left(A^{-1}\right)^{*} A^{-1}=\left(A^{*}\right)^{-1} A^{-1}=\left(A A^{*}\right)^{-1}=I
$$

showing that $A^{-1}$ is again unitary.

Thus, the collection of unitary matrices is a subgroup of $G L(n, C)$. We call this group the unitary group and we denote it by $U(n)$. We may also define the special unitary group $S U(n)$, the subgroup of $U(n)$ consisting of unitary matrices with determinant 1 . It is easy to check that both $U(n)$ and $S U(n)$ are closed subgroups of $G L(n, C)$ and thus matrix Lie groups.

Meanwhile, let $\langle.,$.$\rangle denote the standard inner product on C^{n}$, given by

$$
\langle x, y\rangle=\sum_{j} \overline{x_{j}} y_{j}
$$

(Note that we put the conjugate on the first factor in the inner product.)

We have

$$
\langle x, A y\rangle=\left\langle A^{*} x, y\right\rangle
$$

for all $x, y \in C^{n}$. Thus

$$
\langle A x, A y\rangle=\left\langle A^{*} A x, y\right\rangle
$$

from which we can see that if $A$ is unitary, then $A$ preserves the inner product on $C^{n}$, that is,

$$
\langle A x, A y\rangle=\langle x, y\rangle
$$

for all $x$ and $y$. Conversely, if $A$ preserves the inner product, we must have $\left\langle A^{*} A x, y\right\rangle=\langle x, y\rangle$ for all $x, y$. It is not hard to see that this condition holds only if $A^{*}=I$. Thus, an equivalent characterization of unitarity is that $A$ is unitary if and only if $A$ preserves the standard inner product on $C^{n}$.

Finally, for any matrix $A$, we have that $\operatorname{det} A^{*}=\overline{\operatorname{det} A}$. Thus, if $A$ is unitary, we have 


$$
\operatorname{det}\left(A^{*} A\right)=|\operatorname{det} A|^{2}=\operatorname{det} I=1
$$

Hence, for all unitary matrices $A$, we have $|\operatorname{det} A|=1$.

In a similar fashion, an $n \times n$ real matrix $A$ is said to be orthogonal if the column vectors of $A$ are orthonormal. As in the unitary case, we may give equivalent versions of this condition. The only difference is that if $A$ is real, $A$ is the same as the transpose $A^{\text {tr }}$ of $A$, given by

$$
\left(A^{t r}\right)_{j k}=A_{k j}
$$

Thus, $A$ is orthogonal if and only if $A^{t r}=A^{-1}$, and this holds if and only if $A$ preserves the inner product on $R^{n}$. Since $\operatorname{det}(A)^{t r}=\operatorname{det} A$, if $A$ is orthogonal, we have

$$
\operatorname{det}\left(A^{\text {tr }} A\right)=\operatorname{det}(A)^{2}=\operatorname{det}(I)=1
$$

so that $\operatorname{det}(A)= \pm 1$. The collection of all orthogonal matrices forms a closed subgroup of $G L(n, C)$. which we call the orthogonal group and denote by $O(n)$.

The set of $n \times n$ orthogonal matrices with determinant one is the special orthogonal group, denoted $S O(n)$. Geometrically, elements of $S O(n)$. are rotations, while the elements of $O(n)$. are either rotations or combinations of rotations and reflections. Consider now the bilinear form (...) on $C^{n}$ defined by

$$
(x, y)=\sum_{j} x_{j} y_{j}
$$

This form is not an inner product because, for example, it is symmetric rather than conjugate symmetric. The set of all $n \times n$ complex matrices $A$ which preserve this form (i.e., such that $(A x, A y)=(x, y)$ for all $\left.x, y \in C^{n}\right)$ is the complex orthogonal group $O(n ; C)$, and it is a subgroup of $G L(n ; C)$. Since there are no conjugates in the definition of the form (...), we have

$$
(x, A y)=\left(A^{t r} x, y\right)
$$

for all $x, y \in C^{n}$ where on the right-hand side of the above relation, we have $A^{\text {tr }}$ rather than $A^{*}$. Repeating the arguments for the case of $O(n)$, but now allowing complex entries in our matrices, we find that an $n \times n$ complex matrix $A$ is in $O(n ; C)$, if and only if $A^{t r} A=I$, that $O(n ; C)$, is a matrix Lie group, and that $\operatorname{det}(A)= \pm 1$ for all $A$ in $O(n ; C)$. Note that $O(n ; C)$ is not the same as the unitary group $U(n)$.

The group $S O(n ; C)$ is defined to be the set of all $A$ in $O(n ; C)$ with $\operatorname{det}(A)=1$ and it is also a matrix Lie group.

\section{[5] Symplectic Groups}

Consider the skew-symmetric bilinear form B on $R^{2 n}$ defined as follows

$$
\omega(x, y)=\sum_{j=1}^{n}\left(x_{j} y_{n+j}-x_{n+j} y_{n}\right)
$$

The set of all $2 n \times 2 n$ real matrices A which preserve $\omega$ (i.e., such that 
$\omega(A x, A y)=\omega(x, y)$ for all $\left.x, y \in R^{2 n}\right)$ is the real symplectic group $\operatorname{Sp}(n, R)$, and it is a closed subgroup of $G L(2 n, R)$. (Some authors refer to the group we have just defined as $\operatorname{Sp}(2 n, R)$ rather than $S p(n, R)$ If is the $2 n \times 2 n$ matrix

$$
\Omega=\left(\begin{array}{cc}
0 & I \\
-I & 0
\end{array}\right)
$$

Then

$$
\omega(x, y)=\langle x, \Omega y\rangle
$$

From this, it is not hard to show that a $2 n \times 2 n$ real matrix $A$ belongs to $\operatorname{Sp}(n, R)$ if and only if

$$
-\Omega A^{t r} \Omega=A^{-1}
$$

Taking the determinant of this identity gives $\operatorname{det} A=(\operatorname{det} A)^{-1}$, i.e. $\operatorname{det}(A)^{2}=1$. This shows that $\operatorname{det} A= \pm 1$, for all $A \in S p(n, R)$. In fact, $\operatorname{det} A=1$ for all $A \in \operatorname{Sp}(n, R)$, although this is not obvious. One can define a bilinear form $\omega$ on $C^{2 n}$ by the same formula as in

$$
\omega(x, y)=\sum_{j=1}^{n}\left(x_{j} y_{n+j}-x_{n+j} y_{n}\right)
$$

(with no conjugates).

Over $C$, we have the relation

$$
\omega(z, w)=(z, \Omega w)
$$

where (.,.) is the complex bilinear form in $(x, y)=\sum_{j} x_{j} y_{j}$. The set of $2 n \times 2 n$ complex matrices which preserve this form is the complex symplectic group $S p(n, C)$. A $2 n \times 2 n$ complex matrix $A$ is in $S p(n, C)$ if and only if $-\Omega A^{\text {tr }} \Omega=A^{-1}$ holds. (Note: This condition involves $A^{\text {tr }}$ not $A$.) Again, we can easily show that each $A \in \operatorname{Sp}(n, C)$. Satisfies $\operatorname{det} A=1$ and, again, it is actually the case that $\operatorname{det} A=1$. Finally, we have the compact symplectic group $\operatorname{Sp}(n)$ defined as

$$
S p(n)=S p(n, C) \cap U(2 n)
$$

[6]: The groups $R^{*}, C^{*}, S^{1}$ and $R^{n}$

The groups $R^{*}$ and $C^{*}$ under matrix multiplication are isomorphic to $G L(1, R)$ and $G L(1, C)$, respectively, and so we view them as matrix Lie groups. The group $S^{1}$ of complex numbers with absolute value one is isomorphic to $U(1)$ and so we also view it as a matrix Lie group. The group $R^{n}$ under vector addition is isomorphic to the group of diagonal real matrices with positive diagonal entries, via the map

$$
\left(\begin{array}{lll}
x_{1} & \cdots & x_{n}
\end{array}\right) \rightarrow\left(\begin{array}{ccc}
\mathrm{e}^{x_{1}} & & 0 \\
\vdots & \ddots & \vdots \\
0 & & \mathrm{e}^{x_{n}}
\end{array}\right)
$$

One easily checks that this is a matrix Lie group and thus we view $R^{n}$ as a matrix Lie group as well. 
Definition 2. [7] A subgroup $H$ of a Lie group $G$ is called a Lie subgroup if it is a Lie group (with respect to the induced group operation), and the inclusion map $\imath_{H}: H \rightarrow G$ is a smooth immersion (and therefore a Lie group homomorphism).

Example 8. [7] Consider $G=T^{2}=S^{1} \times S^{1}$. Then $S^{1} \times\{0\}$ and $\{0\} \times S^{1}$ are Lie subgroups. Moreover, for any co-prime pair of integers $(p ; q)$,

$$
H^{p, q}=\left\{\left(\mathrm{e}^{i p t}, \mathrm{e}^{i q t}\right) \mid t \in R\right\}
$$

is a Lie subgroup of $T^{2}$. These are submanifolds as well. However, there are also many Lie subgroups of $T^{2}$ which are not submanifolds. In fact, for any irrational number $\alpha$,

$$
H^{\alpha}=\left\{\left(\mathrm{e}^{i t}, \mathrm{e}^{i \alpha t}\right) \mid t \in R\right\}
$$

is a Lie subgroup of $T^{2}$. But $\bar{H}^{\alpha}=T^{2}$, so they are not submanifolds.

Definition 3. A Lie subgroup $H$ of $G$ is said to be a closed Lie subgroup if $H$ is both a Lie subgroup and also a submanifold of $G$.

Lemma 1 [7]. Suppose $G$ is a Lie group, $H$ is a subgroup of $G$ which is a submanifold as well. Then $H$ is closed in the sense of topology.

Proof. Since $H$ is a submanifold of $G$, it is locally closed everywhere. In particular, one can find an open neighborhood $U$ of $e$ in $G$ such that $U \cap H=U \cap \bar{H}$. Now take any $h \in \bar{H}$. Since $h U$ is an open neighborhood of $h$ in $G, h U \cap H \neq \varnothing$. Let $h^{\prime} \in h U \cap H$, then $h^{-1} h^{\prime} \in U$. On the other hand, since $h \in H$, there is a sequence $h_{n}$ in $H$ converging to $h$. It follows that the sequence $h^{-1} h^{\prime} \in H$ converges to $h^{-1} h^{\prime}$.

In other words, $h^{-1} h^{\prime} \in U \cap \bar{H}$. So $h \in H$, i.e. $\bar{H} \subset H$. Therefore, $H$ is closed.

\section{Homomorphisms of Lie Groups}

\section{Lie group homomorphism}

Let $G$ and $H$ be Lie groups. A map $\varphi: G \rightarrow H$ is called a Lie group homomorphism if

1) $\varphi$ is a group homomorphism, and

2) $\varphi$ is continuous.

\section{Lie group isomorphism}

Let $G$ and $H$ be Lie groups. A map $\varphi: G \rightarrow H$ is called a Lie group isomorphism if

1) $\varphi$ is one-to-one and onto, and

2) the inverse map is $\varphi^{-1}$ continuous.

Examples 1 [6]:

1) The map $R \rightarrow U(1)$ given by $\theta \rightarrow \mathrm{e}^{\mathrm{i} \theta}$ is a Lie group homomorphism.

2) The map $U(1) \rightarrow S O(2)$ given by

$$
\mathrm{e}^{i \theta} \rightarrow\left(\begin{array}{cc}
\cos \theta & -\sin \theta \\
\sin \theta & \cos \theta
\end{array}\right)
$$


is a Lie group isomorphism (you should check that this map is well-defined and is indeed an isomorphism).

1) Composing the previous two examples gives the Lie algebra homomorphism $R \rightarrow S O(2)$ defined by

$$
\theta \rightarrow\left(\begin{array}{cc}
\cos \theta & -\sin \theta \\
\sin \theta & \cos \theta
\end{array}\right)
$$

2) The determinant is a Lie group homomorphism $G L(n, C) \rightarrow C^{*}$.

\section{Invariant Vector Fields and the Exponential Map}

Definition 1. A vector field $v \in V(G)$ is left-invariant if $g * v=v$ for every $g \in G$, and right-invariant if $v * g=v$ for every $g \in G$. A vector field is called bi-invariant if it is both left- and right-invariant.

Theorem 1 [8]. The map $v \rightarrow v(1)$ (where 1 is the identity element of the group) defines an isomorphism of the vector space of left-invariant vector fields on $G$ with the vector space $T_{e} G$, and similarly for right-invariant vector spaces. Proof. It suffices to prove that every $x \in T_{e} G$ can be uniquely extended to a left-invariant vector field on $G$. Let us define the extension by $v(g)=g \cdot x \in T_{g} G$. Then one easily sees that the so-defined vector field is left-invariant, and $v(1)=x$. This proves the existence of an extension; uniqueness is obvious.

Definition 2. A smooth homomorphism $\varphi:(R,+) \rightarrow G$ is called a one parameter group subgroup of $G$.

Definition 3. Let $G$ be a real or complex Lie group. Then the exponential map $\exp : T_{e} G \rightarrow G$ is defined by

$$
\exp (x)=\alpha_{x}(1)
$$

where $\alpha_{x}(t)$ is the one-parameter subgroup with tangent vector at 1 equal to $x$.

Example 3 [3]. We return to the example of the group $G L(V)$ with $V$ a finite dimensional real linear space. Its neutral element e equals $I=I_{V}$. Since $G L(V)$ is open in $\operatorname{End}(V)$, we have $\operatorname{TeGL}(V)=\operatorname{End}(V)$. If $x \in G L(V)$ then $l_{x}$ is the restriction of the linear map $L_{x}: A \rightarrow x A ; \operatorname{End}(V) \rightarrow \operatorname{End}(V)$ to $G L(V)$ hence $T_{e}\left(l_{x}\right)=L_{x}$. Hence, the integral curve $\alpha_{x}$ satisfies the equation:

$$
\frac{\mathrm{d}}{\mathrm{d} t} \alpha(t)=\alpha(t) X
$$

Since $t \rightarrow \mathrm{e}^{t X}$ is a solution to this equation with the same initial value, we must have that $\alpha_{X}(t)=\mathrm{e}^{t X}$. Thus in this case exp is the ordinary exponential map $X \rightarrow \mathrm{e}^{X}$, End $(V) \rightarrow G L(V)$.

Proposition 1 [9]. The exponential map $\exp : T_{e} G \rightarrow G$ satisfies:

1) For each $X \in T_{e} G, \alpha(t)=\exp (t X)$ is a one parameter group with $\alpha^{\prime}(0)=X$. 
2) The integral curve $\mathrm{c}$ of the left invariant vector field $X \in T_{e} G$ with $c(0)=T_{e} G$ is

$$
c(t)=T_{e} G \exp (t X) .
$$

3) exp is smooth with $d(\exp )_{0}=I d$.

4) If $\varphi: H \rightarrow G$ is a Lie group homomorphism, then $\varphi\left(\exp _{H}(X)\right)=\exp _{G}(d \varphi(X))$ for $X \in h$.

5) If $H \subset G$ is a Lie subgroup then

$$
h=\left\{X \in T_{e} G \mid \exp _{G}(t X) \in H \text { for }|t|<\varepsilon \text { for some } \varepsilon>0\right\}
$$

Proof: First observe that $\alpha_{X}$ is an integral curve of $X$ through $e$ since

$$
\begin{aligned}
\alpha_{X}^{\prime}(s) & =\frac{\mathrm{d}}{\mathrm{d} t}\left(\alpha_{X}(s+t)\right)_{t=0}=\frac{\mathrm{d}}{\mathrm{d} t}\left(\alpha_{X}(s) \alpha_{X}(t)\right)_{t=0} \\
& =T_{e}\left(L_{\alpha_{X}(s)}\right)_{e}\left(\alpha_{X}^{\prime}(0)\right)=T_{e}\left(L_{\alpha_{X}(s)}\right)_{e}(X)=X\left(\alpha_{X}(s)\right)
\end{aligned}
$$

Thus $\alpha_{t X}(s)=\alpha_{X}(t s)$, since, for fixed $t$, both are integral curves of $t X$ through $e$. To see this for the right hand side, observe that in general if $\gamma(s)$ is an integral curve of a vector field $X$, then $\gamma(t s)$ is an integral curve of $t X$. Hence $\exp (t X)=\alpha_{t X}(1)=\alpha_{X}(t)$, which implies (a). Since $L_{T_{e} G}$ takes integral curves to integral curves, (b) follows as well.

To see that exp is smooth, define a vector field $Z$ on $G \times T_{e} G$ by $Z\left(T_{e} G, X\right)=\left(X\left(T_{e} G\right), 0\right) . Z$ is clearly smooth and by part (b), its flow is $\gamma_{t}\left(T_{e} G, X\right)=(\exp (t X), X)$. Thus $\gamma_{1}(e, X)=(\exp (X), X)$ is smooth in $X$ and hence exp is smooth as well. Finally, $d(\exp )_{0}(X)=\frac{\mathrm{d}}{\mathrm{d} t}(\exp (t X))_{t=0}=X$, which proves the second claim in (c).

To prove (d), observe that a homomorphism takes one parameter groups to one parameter groups. Thus $\gamma(t)=\alpha(\exp (t X))$ is a one parameter group with $\gamma^{\prime}(0)=d \alpha\left(d(\exp )_{0}(X)\right)=\alpha(X)$ and hence $\alpha(t)=\exp (t d \alpha(X))$, which proves our claim by setting $t=1$.

Part (e) follows easily by applying (d) to the inclusion of $H$ in $G$.

Example 4. $G L(n ; R)$ and $G L(n ; C)$, the set of invertible matrices, are Lie groups. For these groups we claim that $\exp (A)=\mathrm{e}^{A}$, which explains the name exponential map. Indeed, from the power series definition of $\mathrm{e}^{A}$ it easily follows that $\mathrm{e}^{(t+s) A}=\mathrm{e}^{t A} \mathrm{e}^{s A}$, i.e. $\alpha(t)=\mathrm{e}^{t A}$ is a one parameter group. Furthermore $\alpha^{\prime}(0)=A$ and hence $\exp (A)=\alpha(1)=\mathrm{e}^{A}$.

Lemma 1 [3]: Let $\varphi: G \rightarrow H$ be a homomorphism of Lie groups. Then the following diagram commutes

$$
\begin{array}{rll}
G & \stackrel{\varphi}{\rightarrow} & H \\
\exp _{G} \uparrow & & \uparrow \\
T_{e} G & \stackrel{T_{e} \varphi}{\rightarrow} & T_{e} H
\end{array}
$$

Proof: Let $X \in T_{e} G$. Then $\alpha(t)=\varphi\left(\exp _{G}(t X)\right)$ is a one-parameter subgroup of $H$ : Differentiating at $t=0$ we obtain $\dot{\alpha}(0)=T_{e}(\varphi) T_{0}\left(\exp _{G}\right) X=T_{e}(\varphi) X$. Now apply the above lemma to conclude that $\alpha(t)=\exp _{H}\left(t T_{e}(\varphi) X\right)$. The re- 
sult follows by specializing to $t=1$.

\section{The Lie Algebra of a Lie Group}

For $x \in G$ consider the conjugation map $C_{x}=l_{x} \circ r_{x^{-1}}$. Since $C_{x}$ is a homomorphism

$$
\operatorname{Ad}(x)=T_{e} C_{x}
$$

is a Lie algebra homomorphism.

The map Ad:G $\rightarrow G L\left(T_{e} G\right)$ is called the adjoint representation of $G$ in $T_{e} G$.

For $X \in T_{e} G$ let $a d X: T_{e} G \rightarrow T_{e} G$ be defined by $a d_{X}(Y)=[X, Y]$. The Jacobi identity is equivalent to saying that

$$
\begin{aligned}
a d_{[X, Y]}=a d_{X} a d_{Y}-a d_{Y} a d_{X}= & {\left[a d_{X}, a d_{Y}\right] \text { i.e. } } \\
& a d: T_{e} G \rightarrow \operatorname{End}\left(T_{e} G\right)
\end{aligned}
$$

is defined by

$$
a d=T_{e} A d
$$

We note that, by the chain rule, for all $X \in T_{e} G$;

$$
\operatorname{ad}(X)=\left.\frac{\mathrm{d}}{\mathrm{d} t}\right|_{t=0} \operatorname{Ad}(\exp t X)
$$

Proposition [5]. If $g \rightarrow T_{e} G$ is a Lie algebra, then

$$
a d_{[X, Y]}=a d_{X} a d_{Y}-a d_{Y} a d_{X}=\left[a d_{X}, a d_{Y}\right]
$$

that is, ad $: T_{e} G \rightarrow \operatorname{End}\left(T_{e} G\right)$ is a Lie algebra homomorphism.

Proof. Observe that

$$
\operatorname{ad}_{[X, Y]}(Z)=[[X, Y], Z]
$$

whereas

$$
\left[a d_{X}, a d_{Y}\right](Z)=[X,[Y, Z]]-[Y,[X, Z]]
$$

Thus, we want to show that

$$
[[X, Y], Z]=[X,[Y, Z]]-[Y,[X, Z]]
$$

which is equivalent to the Jacobi identity

Lemma 1: The adjoint representation satisfies:

1) $T_{e}(\operatorname{Ad}(X))=a d_{X}$ or simply $T_{e} A d=a d$

2) $\operatorname{Ad}(\exp (X))=\mathrm{e}^{a d_{X}}$

Proof: For part a) we see that for any $Y \in T_{e} G$

$$
\begin{aligned}
& T_{e}(\operatorname{Ad}(X))(Y)=\left.\frac{\mathrm{d}}{\mathrm{d} t}\right|_{t=0} \operatorname{Ad}(\exp (t X))(Y) \\
& =\left.\frac{\mathrm{d}}{\mathrm{d} t}\right|_{t=0} T_{e}\left(R_{\exp (-t X)}\right) \circ T_{e}\left(L_{\exp (t X)}\right)(Y) \\
& =\left.\frac{\mathrm{d}}{\mathrm{d} t}\right|_{t=0} T_{e}\left(R_{\exp (-t X)}\right)(Y(\exp (t X)))
\end{aligned}
$$




$$
L_{X} Y=[X, Y]
$$

where $L_{X}$ is the Lie derivative. In the last passage, we used the definition of Lie derivative, and the fact that $R_{\exp (t X)}$ is the flow of $X$.

We may apply Lemma 1 with $H=G L\left(T_{e} G\right)$ and $\varphi=A d$. Since

$T_{e} H=T_{I} G L\left(T_{e} G\right)=\operatorname{End}\left(T_{e} G\right)$ whereas $\exp _{H}$ is given by $X \rightarrow \mathrm{e}^{X}$; we see that the following diagram commutes:

$$
\begin{array}{ccc}
G & \stackrel{A d}{\rightarrow} & G L\left(T_{e} G\right) \\
\exp \uparrow & & \uparrow \\
T_{e} G & \stackrel{\text { ad }}{\rightarrow} & \operatorname{End}\left(T_{e} G\right)
\end{array}
$$

Example 1. [3] Let $V$ be finite dimensional real linear space. Then for $x \in G L(V)$ the linear map $\operatorname{Ad}(x): \operatorname{End}(V) \rightarrow \operatorname{End}(V)$ is given by $A d(x) Y=x Y x^{1}$ : Substituting $x=\mathrm{e}^{t X}$ and differentiating the resulting expression with respect to $t$ at $t=0$ we obtain:

$$
(\operatorname{adX}) Y=\left.\frac{\mathrm{d}}{\mathrm{d} t}\left[\mathrm{e}^{t X} Y \mathrm{e}^{-t X}\right]\right|_{t=0}=X Y-Y X
$$

Hence in this case. $(a d X) Y$ is the commutator bracket of $X$ and $Y$.

Definition 3. For $X, Y \in T_{e} G$ we define the Lie bracket $[X, Y] \in T_{e} G$ by

$$
[X, Y]=(a d X) Y
$$

Example 2. Let $X$ and $Y$ be $n \times n$ matrices. Show by induction that

$$
\left(a d_{X}\right)^{m}(Y)=\sum_{i=0}^{m}\left(\begin{array}{c}
m \\
i
\end{array}\right) X^{i} Y(-X)^{m-i}
$$

where

$$
\left(a d_{X}\right)^{m}(Y)=[\underbrace{X, \cdots[X,[X, Y]] \cdots}_{m}]
$$

Now, show by direct computation that

$$
\mathrm{e}^{a d_{X}}(Y)=A d_{e} x(Y)=\mathrm{e}^{X} Y \mathrm{e}^{-X}
$$

Lemma 1. [3] Let $\varphi: G \rightarrow H$ be a homomorphism of Lie groups. Then the following diagram commutes:

$$
T_{e} \varphi\left([X, Y]_{G}\right)=\left[T_{e} \varphi X, T_{e} \varphi Y,\right]_{H}\left(X, Y \in T_{e} G\right)
$$

Proof: One readily verifies that $\varphi \circ C_{x}^{G}=C_{\varphi(x)}^{H} \circ \varphi$. Taking the tangent map of both sides of this equation at $e$, we obtain that the following diagram commutes:

$$
\begin{array}{rlrl}
T_{e} G & \stackrel{T_{e} \varphi}{\rightarrow} & T_{e} H \\
A d_{G}(x) \uparrow & & \uparrow & d_{H}(\varphi(x)) \\
T_{e} G & \stackrel{T_{e} \varphi}{\rightarrow} & T_{e} H
\end{array}
$$

Differentiating once more at $x D e$, in the direction of $X \in T_{e} G$; we obtain that the following diagram commutes: 


$$
\begin{array}{cccc}
T_{e} G & \stackrel{T_{e} \varphi}{\rightarrow} & T_{e} H \\
a d_{G}(X) \uparrow & & \uparrow a d_{H}\left(T_{e} \varphi X\right) \\
T_{e} G & \stackrel{T_{e} \varphi}{\rightarrow} & T_{e} H
\end{array}
$$

We now agree to write $[X, Y]=(\operatorname{ad} X) Y$.

\section{The Future Perspective of This Paper}

The future perspective of this paper is that since this is a work of a general nature, it is necessary to extract the most important things from it. It is possible to do a paper for each of the titles in the paper. Each of the titles creates an opportunity for research because from each title there are many opportunities to explore and write. It is my future to work on comparing many facts and the application of leftist groups in everyday life. To give a glimpse of a better tomorrow of this beautiful science called both left groups and left algebras and their close connection to other mathematical disciplines primarily thinking of linear algebra, geometry, analysis and topology. Comparing all these disciplines with the left groups, we see a close connection and the need to apply and use them in the right way.

\section{Conflicts of Interest}

The author declares no conflicts of interest regarding the publication of this paper.

\section{References}

[1] Bellamy, G. (2016) Lie Groups, Lie Algebras, and Their Representations. http://www.maths.gla.ac.uk/ gbellamy/lie.pdf

[2] Renee, A. (2017) An Introduction to Lie Algebra. Talley California State University, San Bernardino.

https://scholarworks.lib.csusb.edu/cgi/viewcontent.cgi?article=1668\&context=etd

[3] Van den Ban, E.P. (2010) Lie Groups, Lecture Notes. https://webspace.science.uu.nl/ ban00101/lecnotes/lie2010.pdf

[4] Study Document-Trinity College Dublin (2015) Linear Groups. https://www.maths.tcd.ie/pub/coursework/424/GpReps-IV.pdf

[5] Hall, B.C. (2015) Lie Groups, Lie Algebras, and Representations. Department of Mathematics, University of Notre Dame, Notre Dame, USA.

[6] Savage, A. (2015) Introduction to Lie Groups. Department of Mathematics and Statistics University of Ottawa, Ottawa. https://alistairsavage.ca/mat4144/notes/MAT4144-5158-LieGroups.pdf

[7] Wang, Z.Q. (2010) Lie Subgroups v.s. Lie Subalgebras. http://staff.ustc.edu.cn/ wangzuoq/Courses/13F-Lie/Notes/Lec\%2010.pdf

[8] Kirillovan Jr., A. (2008) Introduction to Lie Groups and Lie Algebras. Cambridge University Press, Cambridge. http://www.cambridge.org/9780521889698 http://www.cambridge.org

[9] Ziller, W. (2010) Lie Groups. Representation Theory and Symmetric Spaces. University of Pennsylvania, Philadelphia.

https://www.math.upenn.edu/ wziller/math650/LieGroupsReps.pdf 